Pacific

Journal of

Mathematics

\title{
ON COMMENSURABILITY OF FIBRATIONS ON
}

A HYPERBOLIC 3-MANIFOLD

HideTOSHI MASAI 


\title{
ON COMMENSURABILITY OF FIBRATIONS ON A HYPERBOLIC 3-MANIFOLD
}

\author{
Hidetoshi MASAI
}

\begin{abstract}
We discuss fibered commensurability of fibrations on hyperbolic 3-manifolds, a notion introduced by Calegari, Sun, and Wang (Pacific J. Math. 250:2 (2011), 287-317). We construct manifolds with nonsymmetric but commensurable fibrations on the same fibered face, and prove that if a given manifold $M$ does not have hidden symmetries, then $M$ does not admit nonsymmetric but commensurable fibrations.

It was also proved by Calegari et al that every hyperbolic fibered commensurability class contains a unique minimal element. Here we provide a detailed discussion on the proof of the theorem in the cusped case.
\end{abstract}

\section{Introduction}

In this paper, we are mainly interested in fibered hyperbolic 3-manifolds with the first Betti number greater than or equal to 2. Thurston [1986] showed that such a manifold admits infinitely many distinct fibrations (see also Section 4). It is an interesting question to investigate the relationship between such fibrations.

Calegari, Sun, and Wang defined the notion of fibered commensurability, which gives rise to an equivalence relation on fibrations. An automorphism on a surface is an isotopy class of self-homeomorphisms of the surface. For any fibration on a 3-manifold, we have the pair $(F, \phi)$ of the fiber surface $F$, and the monodromy automorphism $\phi$. Since the monodromy is determined up to conjugacy in the mapping class group of $F$, we use the notation $(F, \phi)$ to denote the conjugacy class. Then commensurability of fibrations is defined as follows.

Definition 1.1 [Calegari et al. 2011]. A pair $(\tilde{F}, \tilde{\phi})$ covers $(F, \phi)$ if there is a finite cover $\pi: \widetilde{F} \rightarrow F$ and representative homeomorphisms $\tilde{f}$ of $\tilde{\phi}$ and $f$ of $\phi$ so that $\pi \tilde{f}=f \pi$ as maps $\widetilde{F} \rightarrow F$.

Definition 1.2 [Calegari et al. 2011]. Two pairs $\left(F_{1}, \phi_{1}\right)$ and $\left(F_{2}, \phi_{2}\right)$ are commensurable if there is a surface $\widetilde{F}$, automorphisms $\widetilde{\phi}_{1}$ and $\widetilde{\phi}_{2}$, and nonzero integers $k_{1}$

This work was partially supported by JSPS Research Fellowship for Young Scientists.

MSC2010: primary 57M50; secondary 37B40.

Keywords: hyperbolic manifold, fibration, commensurability. 
and $k_{2}$, so that $\left(\widetilde{F}, \tilde{\phi}_{i}\right)$ covers $\left(F_{i}, \phi_{i}\right)$ for $i=1,2$ and if $\tilde{\phi}_{1}^{k_{1}}=\tilde{\phi}_{2}^{k_{2}}$ as automorphisms of $\widetilde{F}$.

For the remainder of the paper, we consider fibrations on hyperbolic 3-manifolds. In this case, the monodromy of each fibration is always pseudo-Anosov (see Definition 2.5 for the definition). The normalized entropy of a conjugacy class $(F, \phi)$ is defined as $\chi(F) \log \lambda(\phi)$, where $\chi(F)$ is the Euler characteristic of $F$ and $\lambda(\phi)$ is the dilatation of $\phi$. In Section 2, we observe that the normalized entropies of commensurable fibrations on the same hyperbolic 3-manifold agree. Then we offer an example of a manifold such that two of its fibrations are commensurable if and only if they share the same normalized entropy. We also give an example of a manifold with two noncommensurable fibrations of the same normalized entropy.

In this paper, we study commensurable fibrations on a hyperbolic 3-manifold in the context of a fibered face. A fibered face is a face of the Thurston norm ball whose rational points correspond to fibrations of the 3-manifold and a fibered cone is a cone over a fibered face (see Section 3 for details). Two fibrations on $M$ are said to be symmetric if there exists a self-homeomorphism $\varphi: M \rightarrow M$ that maps one to the other. In [Calegari et al. 2011, Remark 3.9], Calegari, Sun, and Wang asked if there is an example of two fibrations on the same closed manifold, which are commensurable but have fibers distinguished by their genera. The following theorem provides such a construction in the cusped case. In this theorem fibers are distinguished by their Euler characteristics (see Section 4 for a proof).

Theorem 1.3. There are hyperbolic 3-manifolds with nonsymmetric but commensurable fibrations whose corresponding elements in $H^{1}(M ; \mathbb{Z})$ are in the same fibered cone.

On the other hand, if $M$ has no hidden symmetries, then such fibrations do not exist. Here, a (finite-volume) hyperbolic 3-manifold $M=\mathbb{H}^{3} / \Gamma$ is said to have hidden symmetries if $\left[C^{+}(\Gamma): N^{+}(\Gamma)\right]>1$, where $C^{+}(\Gamma)$ and $N^{+}(\Gamma)$ are the commensurator and normalizer of $\Gamma$; see Section 4 for details.

Theorem 1.4. Suppose that $M$ is a hyperbolic 3-manifold that does not have hidden symmetries. Then, any pair of fibrations of $M$ is either symmetric or noncommensurable, but not both.

Theorems 1.3 and 1.4 are motivated by the fact that up to isotopy, there are only finitely many commensurable fibrations on a hyperbolic 3 -manifold. This fact is a corollary of the following:

Theorem 1.5 (see also Theorem 3.1 of [Calegari et al. 2011]). Every commensurability class of hyperbolic fibered pairs contains a unique (orbifold) minimal element. 
Here the notion of a fibered pair is a generalization of the notion of a pair $(F, \phi)$, see Section 2 for details. The proof in [Calegari et al. 2011] works for the closed case. In Section 2 we extend it to the case where the manifolds have boundary (Theorem 2.6). Further, as a corollary of this extension, we show examples of manifolds such that every fibration is the minimal element in its commensurability class (Corollary 2.8).

Commensurability classes are defined using the transitive hull of the relation in Definition 1.2. In Section 2 we also discuss the transitivity of commensurability. We show that if the automorphisms are pseudo-Anosov (that is to say, in the hyperbolic case), then commensurability is transitive.

\section{Preliminaries}

In this section, we recall the definitions and basic facts about commensurability of fibrations. Most of the contents in this section are discussed in [Calegari et al. 2011]. In this paper, unless otherwise stated, by a surface and a hyperbolic 3-manifold, we mean a compact connected orientable 2-manifold possibly with boundary and of negative Euler characteristic, and a connected, orientable, complete hyperbolic 3-manifold of finite volume respectively.

Fibered pairs. Given a homeomorphism $f: F \rightarrow F$, the mapping torus of $f$ is the 3-manifold

$$
M=F \times[0,1] /((f(x), 0) \sim(x, 1)) .
$$

Mapping tori of conjugate automorphisms are homeomorphic, so if $\phi$ is a conjugacy class of homeomorphisms we obtain a homeomorphism class of mapping tori, which we denote by $[F, \phi]$. We call $F$ the fiber and $\phi$ the monodromy of $[F, \phi]$.

We will focus on fibrations of a fixed hyperbolic 3-manifold $M$. Each fibration on $M$ over the circle determines an element of $H^{1}(M ; \mathbb{Z})$, and if $\omega \in H^{1}(M ; \mathbb{Z})$ corresponds to a fibration, then there is an associated pair $(F, \phi)$, in the sense that $[F, \phi]$ is homeomorphic to $M$. This correspondence of $\omega$ and $(F, \phi)$ is well defined up to the conjugation of $(F, \phi)$.

Later in this section (page 317), we discuss Theorem 3.1 of [Calegari et al. 2011] for the case of fibered manifolds with boundary. To state the theorem it is convenient to define a fibered pair which is a generalization of a pair of type $(F, \phi)$. We also enlarge our attention to orbifolds. An $n$-orbifold is a space that is locally modeled on a quotient of an open ball in $\mathbb{R}^{n}$ by a finite group. See [Walsh 2011] and Chapter 13 of [Thurston 1979] for more details.

Definition 2.1 [Calegari et al. 2011]. A fibered pair is a pair $(M, \mathscr{F})$, where $M$ is a compact 3-manifold with boundary a union of tori and Klein bottles, and $\mathscr{F}$ is a foliation by compact surfaces. More generally, an orbifold fibered pair is a 
pair $(O, \mathscr{G})$, where $O$ is a compact 3-orbifold, and $\mathscr{G}$ is a foliation of $O$ by compact 2-orbifolds.

Definition 2.2 [Calegari et al. 2011]. A fibered pair $(\tilde{M}, \widetilde{\mathscr{F}})$ covers $(M, \mathscr{F})$ if there is a finite covering of manifolds $\pi: \tilde{M} \rightarrow M$ such that $\pi^{-1}(\mathscr{F})$ is isotopic to $\widetilde{\mathscr{F}}$. Two fibered pairs $\left(M_{1}, \mathscr{F}_{1}\right)$ and $\left(M_{2}, \mathscr{F}_{2}\right)$ are (fibered) commensurable if there is a third fibered pair $(\tilde{M}, \tilde{\mathscr{F}})$ that covers both.

For a given pair $(F, \phi)$, the mapping torus $[F, \phi]$ has a foliation $\mathscr{F}$ by surface leaves, which are homeomorphic to $F$ and hence there is a corresponding fibered pair $([F, \phi], \mathscr{F})$.

Unlike the case of commensurability in Definition 1.2, it is easy to see that commensurability of fibered pairs is transitive. Suppose $\left(M_{i}, \mathscr{F}_{i}\right)$ and $\left(M_{i+1}, \mathscr{F}_{i+1}\right)$ are commensurable for $i=1,2$ and $\left(\tilde{M}_{12}, \widetilde{\mathscr{F}}_{12}\right)\left(\operatorname{resp} .\left(\tilde{M}_{23}, \widetilde{\mathscr{F}}_{23}\right)\right)$ is a common covering pair of $\left(M_{1}, \mathscr{F}_{1}\right)$ and $\left(M_{2}, \mathscr{F}_{2}\right)\left(\operatorname{resp} .\left(M_{2}, \mathscr{F}_{2}\right)\right.$ and $\left.\left(M_{3}, \mathscr{F}_{3}\right)\right)$. Then there is a covering $p: \tilde{N} \rightarrow M_{2}$ that corresponds to $p_{*}^{12} \pi_{1}\left(\tilde{M}_{12}\right) \cap p_{*}^{23} \pi_{1}\left(\tilde{M}_{23}\right)<\pi_{1}\left(M_{2}\right)$, where $p^{12}: \tilde{M}_{12} \rightarrow M_{2}$ and $p^{23}: \tilde{M}_{23} \rightarrow M_{2}$ are the covering maps. Then $\left(\tilde{N}, p^{-1}\left(\mathscr{F}_{2}\right)\right)$ covers both $\left(M_{1}, \mathscr{F}_{1}\right)$ and $\left(M_{3}, \mathscr{F}_{3}\right)$. Thus we see that fibered commensurability is a transitive relation.

We define another equivalence relation on fibered pairs so that the covering relation will be a partial order.

Definition 2.3 [Calegari et al. 2011]. We say that two fibered pairs $(M, \mathscr{F})$ and $(N, \mathscr{G})$ are covering equivalent if each covers the other. We call a covering equivalence class minimal if no representative covers any element of another covering equivalence class.

Remark 2.4 (see also Remark 2.9 of [Calegari et al. 2011]). Each covering equivalence class of the fibered pair associated to $(F, \phi)$ contains exactly one fibered pair unless $\phi$ is periodic. Therefore, when we consider pseudo-Anosov automorphisms, by abusing notation, we use the word "element" for each covering equivalent class.

Pseudo-Anosov automorphisms. The automorphisms on a compact surface are classified into three types: periodic, reducible, and pseudo-Anosov [Thurston 1988; Casson and Bleiler 1988]. By a result of Thurston, the (interior of the) mapping torus $[F, \phi]$ admits a hyperbolic metric of finite volume if and only if the automorphism $\phi$ is pseudo-Anosov (see [Thurston 1988], and compare [Otal 1996]).

Definition 2.5. A homeomorphism $f: F \rightarrow F$ is a pseudo-Anosov homeomorphism if there is a pair of transverse measured singular foliations $\left(\mathscr{F}^{s}, \mu^{s}\right)$ and $\left(\mathscr{F}^{u}, \mu^{u}\right)$ on $F$ and a positive real number $\lambda$ so that $f\left(\mathscr{F}^{u}\right)=\mathscr{F}^{u}, f\left(\mu^{u}\right)=\lambda \mu^{u}$ and $f\left(\mathscr{F}^{s}\right)=$ $\mathscr{F}^{s}, f\left(\mu^{s}\right)=(1 / \lambda) \mu^{s}$. We call $\left(\mathscr{F}^{s}, \mu^{s}\right)$ and $\left(\mathscr{F}^{u}, \mu^{u}\right)$ the stable and unstable measured singular foliations associated to $f$. 


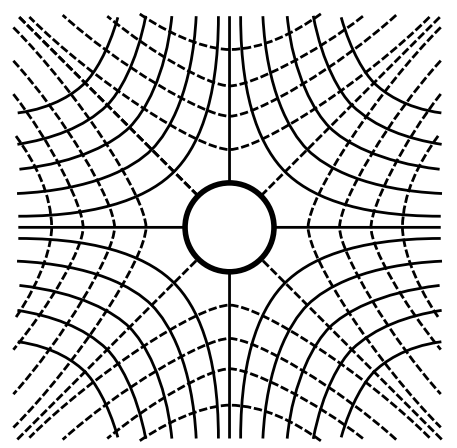

Figure 1. A shape of a singularity of degree 4 at the boundary.

See Figure 1 for a shape of the singularities of $\left(\mathscr{F}^{s}, \mu^{s}\right)$ and $\left(\mathscr{F}^{u}, \mu^{u}\right)$. An automorphism $\phi$ is said to be pseudo-Anosov if it has a pseudo-Anosov homeomorphism as a representative. We call the positive real number $\lambda$ the dilatation of pseudo-Anosov automorphism $\phi$ and denote it by $\lambda(\phi)$.

In some cases, it is convenient to consider the restriction of automorphisms on the interior $\operatorname{Int}(F)$ of $F$. By considering $\left.\phi\right|_{\operatorname{Int}(F)}$, we get a pseudo-Anosov automorphism on $\operatorname{Int}(F)$ and by abusing the notation we also denote it by $\phi$. Note that $\operatorname{Int}(F)$ can be regarded as a surface with finitely many punctures, each corresponding to a boundary component of $F$. Then the singularities of $\mathscr{F}^{s}$ and $\mathscr{F F}^{u}$ lie on $\operatorname{Int}(F)$ or the punctures. We denote the set of points and punctures that correspond to the singular points of associated singular foliations by $\operatorname{Sing}(\phi)$.

Uniqueness of the minimal element. In this subsection, we give a detailed discussion of Theorem 1.5 for the case where manifolds have boundary. By passing to a finite covering we may assume $\mathscr{F}$ to be co-orientable and hence $M$ fibers over the circle; that is, $M$ is the mapping torus $[F, \phi]$ of some surface $F$ and pseudo-Anosov map $\phi$. Since we are dealing with commensurability classes, it suffices to discuss the case where the foliations are co-orientable. The proof in [Calegari et al. 2011] assumes that all singular points of the singular foliations associated to $\phi$ lie on the interior of $F$. We prove this result for the case where some of the singular points lie on the boundary. This corresponds to the case where $\operatorname{Sing}(\phi)$ contains some punctures, by restricting the automorphism on the interior $\operatorname{Int}(F)$ of $F$.

Theorem 2.6 (see also [Calegari et al. 2011]). Let (M, F) be a hyperbolic coorientable fibered pair and let $(F, \phi)$ be the pair associated to $(M, \mathscr{F})$. Then the commensurability class of $(M, \mathscr{F})$ contains a unique minimal (orbifold) element. Moreover, if $\operatorname{Int}(F) \cap \operatorname{Sing}(\phi)=\varnothing$, then the minimal element is a manifold.

Proof. First, we recall the argument in [Calegari et al. 2011], since we will need it here. The stable and unstable singular foliations $\mathscr{F}^{s}$ and $\mathscr{F}^{u}$ associated to $\phi$ 
determine a unique singular Sol metric on $\operatorname{Int}(M)$. Pulling back this metric to the universal cover $\pi: \tilde{M} \rightarrow \operatorname{Int}(M), \tilde{M}$ becomes a simply connected singular Sol manifold. Each fiber of $\tilde{M}$ is a singular Euclidean plane. Let $\Lambda$ be the full isometry group of the singular Sol metric. By appealing to the local Sol metric of $\tilde{M}$, it can be verified that each element of $\Lambda$ preserves the foliation by the singular Euclidean planes. Since $\pi_{1}(M)<\Lambda$, we have the covering $(\tilde{M}, \widetilde{\mathscr{F}}) / \pi_{1}(M) \rightarrow(\tilde{M}, \widetilde{\mathscr{F}}) / \Lambda$. We see that for any pair $\left(M^{\prime}, \mathscr{F}^{\prime}\right)$ commensurable with $(M, F)$ the group $\pi_{1}\left(M^{\prime}\right)$ embeds into $\Lambda$ and hence $\left(M^{\prime}, \mathscr{F}^{\prime}\right)$ covers $(\tilde{M}, \widetilde{\mathscr{F}}) / \Lambda$. Thus the theorem will be proved if we establish the following claim.

Claim 2.7. $\Lambda$ is discrete with respect to the compact open topology.

For the proof of this claim, the condition $\operatorname{Sing}(\phi) \subset \operatorname{Int}(F)$ is assumed in [Calegari et al. 2011]. We prove this claim without the assumption. Note that if $\operatorname{Sing}(\phi) \not \subset$ $\operatorname{Int}(F)$, the singular Sol metric is not necessarily complete. Let $\Lambda^{\prime}<\Lambda$ be the subgroup consisting of isometries that preserve each fiber of $\tilde{M}$ setwise. We first prove that the subgroup $\Lambda^{\prime}$ is discrete. Let $S$ be a fiber of $\tilde{M}$ and $\bar{S}$ be its completion with respect to the singular Euclidean metric. We will extend $p=\left.\pi\right|_{S}: S \rightarrow \operatorname{Int}(F)$ to a local isometry $\bar{p}: \bar{S} \rightarrow \operatorname{Int}(F) \cup \operatorname{Sing}(\phi)$. Let $\left\{x_{i}\right\}$ be a Cauchy sequence in $S$. Then $\left\{p\left(x_{i}\right)\right\}$ is a Cauchy sequence in $\operatorname{Int}(F)$ and it converges to either an interior point of $F$ or a point in $\operatorname{Sing}(\phi)$. Since $\bar{S}$ consists of the equivalence classes of Cauchy sequences in $S$, we can define $\bar{p}:\left[\left(x_{i}\right)\right] \mapsto \lim p\left(x_{i}\right)$. Since $p$ is a local isometry, $\bar{p}$ is well defined and a local isometry. Therefore we get $E:=\bar{S} \backslash S=$ $\bar{p}^{-1}(\operatorname{Sing}(\phi))$ for the natural extension $\bar{p}$ of $p$. Any isometry $\varphi: S \rightarrow S$ extends to an isometry $\bar{\varphi}: \bar{S} \rightarrow \bar{S}$ and by construction we get $\bar{\varphi}(E)=E$. Suppose there is a sequence $\left\{\bar{\varphi}_{i}\right\}$ of isometries such that $\bar{\varphi}_{i} \rightarrow$ id. Since the distances between two distinct points in $E$ are bounded from below by a positive constant, for large enough $i, \bar{\varphi}_{i}$ must fix $E$ pointwise. Suppose that $\bar{\varphi}: \bar{S} \rightarrow \bar{S}$ is an isometry which preserves $E$ pointwise. Since $\bar{S}$ is a singular Euclidean plane, we may find two points $e_{1}, e_{2}$ in $E$ which can be joined by a unique geodesic $\gamma$. By appealing to the distance from $e_{1}$ and $e_{2}$, it follows that $\bar{\varphi}$ preserves $\gamma$ pointwise. Note that every isometry on $\bar{S}$ leaves the set of leaves of $p^{-1}\left(\mathscr{F}^{s}\right)$ and $p^{-1}\left(\mathscr{F}^{u}\right)$ invariant. This implies that every leaf that intersects with $\gamma$ is preserved by $\bar{\varphi}$. Let $l$ be one of such leaves. Since $\bar{\varphi}$ is a local isometry of Sol metric, it locally acts as a translation on $\bar{S}$. Therefore $\bar{\varphi}$ fixes $l$ pointwise. Since each leaf of $\mathscr{F}^{s}$ or $\mathscr{F}^{u} u$ is dense in $\operatorname{Int}(F)$, the orbit of $l$ under the action of the deck transformation group associated to $p$ is also dense in $\bar{S}$. Hence $\bar{\varphi}$ is identity on a dense subset of $\bar{S}$ and since it is an isometry, we get $\bar{\varphi}=\mathrm{id}$. Therefore for large enough $i$, we get $\bar{\varphi}_{i}=\mathrm{id}$. This proves the discreteness of $\Lambda^{\prime}$.

The discreteness of the dynamical direction of $\Lambda$ follows from exactly the same argument in [Calegari et al. 2011]. We include the proof for completeness. Note that each isometry $\varphi \in \Lambda$ extends to the metric completion $\bar{M}$ of $\tilde{M}$. We 


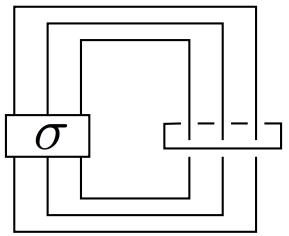

Figure 2. The fibered link associated to a braid $\sigma \in B_{3}$.

may parametrize each fiber by real numbers $t$ in such a way that for any two fixed flow lines $a(t), b(t) \in E(t) \subset \bar{S}(t)$, the distance between $a(t)$ and $b(t)$ is $\sqrt{e^{2 t} x^{2}+e^{-2 t} y^{2}}$ for some fixed $x$ and $y$ when $|t|$ is small enough. For small $|t|$ the distance between any two points in $E(t)$ are bounded from below by a constant which does not depend on $t$. Therefore since $\sqrt{e^{2 t} x^{2}+e^{-2 t} y^{2}}$ is not a locally constant function, an isometry $\varphi \in \Lambda$ close enough to the identity must fix each fiber of the foliation by the singular Euclidean planes. Thus we see that $\Lambda$ is discrete.

Since isometries may fix only singular points, if $\operatorname{Int}(F) \cap \operatorname{Sing}(\phi)=\varnothing$, then $\Lambda$ has no fixed point in $\tilde{M}$ and the last assertion holds.

Corollary 2.8. All the fibrations of $M_{1}=S^{3} \backslash 6_{2}^{2}$ and the magic 3-manifold $M_{2}$ are minimal elements.

Proof. $M_{1}$ (resp. $M_{2}$ ) is homeomorphic to the complement of the fibered link associated to $\sigma_{1} \sigma_{2}^{-1} \in B_{3}$ (resp. $\sigma_{1} \sigma_{2}^{-1} \sigma_{1} \in B_{3}$ ), where $B_{3}$ is the braid group on 3 strands (see Figure 2). It is well known that for every pseudo-Anosov element of $B_{3}$, all singularities are on the punctures. Therefore it suffices to prove that $M_{1}$ and $M_{2}$ are minimal manifolds (not orbifolds) with respect to usual covering relation. $M_{1}$ has volume $4 V_{0}$, where $V_{0} \approx 1.01 \ldots$ is the volume of the ideal regular tetrahedron (see for example [Gehring et al. 1998]). By [Cao and Meyerhoff 2001], $M_{1}$ can only cover the figure-eight knot complements or its sister (m004 or m003 in SnapPea notation). However, SnapPy [Culler et al. 2013] can enumerate all double covers of $\mathrm{m003}$ and $\mathrm{m} 004$ and none of them are homeomorphic to $M_{1}$. Similarly, the magic 3-manifold $M_{2}$ has volume $\approx 5.33 \ldots$ and if it covers a manifold with degree 2 , then its volume is $\approx 2.66 \ldots$, which is less than the volume of the ideal regular octahedron $(\approx 3.66 \ldots)$. By [Agol 2010], such a manifold has only one cusp and cannot be doubly covered by $M_{2}$, which has 3 cusps. Moreover, since $\operatorname{Vol}\left(M_{2}\right) / 3 \approx 1.77 \cdots<2 V_{0}$, again by [Cao and Meyerhoff 2001], $M_{2}$ cannot cover any manifold with degree greater than 2 . Now the result follows from the last assertion of Theorem 2.6.

Remark 2.9. For a fixed surface, there exists a pseudo-Anosov automorphism with the smallest dilatation [Ivanov 1988]. It is interesting to compute the smallest dilatation for a given surface. Hironaka [2010] and Kin and Takasawa [2011] computed 
dilatations of the monodromy of each fiber of $S^{3} \backslash 6_{2}^{2}$ and the magic 3-manifold respectively. It turns out that many small dilatation pseudo-Anosov automorphisms appear as the monodromies of fibrations of those manifolds. Corollary 2.8 shows that all such fibrations are minimal and hence their monodromies can be candidates for the smallest dilatation pseudo-Anosov maps.

Transitivity of commensurability in Definition 1.2. In this subsection, we discuss the subtle difference between fibered commensurability and commensurability in the sense of Definition 1.2. Here, two pairs of type $(F, \phi)$ are said to be fibered commensurable if associated fibered pairs are commensurable. It is easy to see that if two pairs $\left(F_{1}, \phi_{1}\right)$ and $\left(F_{2}, \phi_{2}\right)$ are fibered commensurable, they are commensurable in the sense of following definition.

Definition 2.10 [Carlson 2010]. Two pairs $\left(F_{1}, \phi_{1}\right)$ and $\left(F_{2}, \phi_{2}\right)$ are commensurable if there is a surface $\widetilde{F}$, an automorphism $\widetilde{\phi}$, and nonzero integers $k_{1}$ and $k_{2}$, so that $(\tilde{F}, \tilde{\phi})$ covers $\left(F_{i}, \phi_{i}^{k_{i}}\right)$ for $i=1,2$.

In [Calegari et al. 2011], it is claimed without proof that two pairs $\left(F_{1}, \phi_{1}\right)$ and $\left(F_{2}, \phi_{2}\right)$ are fibered commensurable if and only if they are commensurable in the sense of Definition 1.2. Since a map cannot always be lifted even if a power of it can be lifted, the claim is not trivial. The claim would follow from the transitivity of commensurability in the sense of Definition 1.2, because taking powers of an automorphism is tantamount to a covering. In this subsection, we will prove that the transitivity of commensurability in Definition 1.2 is valid if the automorphisms are pseudo-Anosov.

Proposition 2.11. Suppose that $\left(F_{i}, \phi_{i}\right)$ and $\left(F_{i+1}, \phi_{i+1}\right)$ are commensurable in the sense of Definition 1.2 for $i=1,2$. Suppose further that $\phi_{i}$ are pseudo-Anosov for $i=1,2,3$. Then there exists a pair $\left(F_{123}, \widetilde{\phi}_{i}\right)$ that covers $\left(F_{i}, \phi_{i}\right)$ for each $i=1,2,3$ such that $\widetilde{\phi}_{1}^{k_{1}}=\widetilde{\phi}_{2}^{k_{2}}=\widetilde{\phi}_{3}^{k_{3}}$ for some $k_{1}, k_{2}, k_{3} \in \mathbb{Z} \backslash\{0\}$. In particular, commensurability in the sense of Definition 1.2 is transitive.

Proof. In Theorem 2.6 we proved that each hyperbolic fibered commensurability class contains a unique minimal element. Let $M=\left[F_{1}, \phi_{1}\right]$. Recall that $\Lambda$ is the group of isometries of the singular Sol metric on the universal cover $\tilde{M}$ (see the proof of Theorem 2.6). By considering the subgroup $\Lambda^{+}$that consists of isometries which preserve the orientation of $\widetilde{M}$ and the orientation of the leaf space of $\tilde{M}$. By taking $M_{\text {min }}^{+}:=\tilde{M} / \Lambda^{+}$, we get a unique minimal element among all commensurable fibered pairs both orientable and co-orientable. Although there is a natural extension of this proof in the case where $\tilde{M} / \Gamma^{+}$is an orbifold, such a proof would require more terminology and could obfuscate the key ideas of the proof. Therefore, we only present the case where $\tilde{M} / \Gamma^{+}$is a manifold. In this case we get an associated pair $\left(F_{\min }, \phi_{\min }\right)$ since $M_{\min }^{+}$is orientable and co-orientable. Each 
$\left(F_{i}, \phi_{i}\right)$ covers $\left(F_{\min }, \phi_{\min }^{l_{i}}\right)$ for some $l_{i} \in \mathbb{Z} \backslash\{0\}(i=1,2,3)$. Note that $\phi_{\min }$ is not always lifted to $F_{i}$. Let $H_{i}<\pi_{1}\left(F_{\min }\right)$ be a subgroup which is the image of $\pi_{1}\left(F_{i}\right)$ by the covering map for each $i=1,2,3$. Further let $d=\left[\pi_{1}\left(F_{\min }\right): H_{1} \cap H_{2} \cap H_{3}\right]$, and take $H_{123}:=\bigcap\left\{H<\pi_{1}\left(F_{\min }\right) \mid\left[\pi_{1}\left(F_{\min }\right): H\right]=d\right\}$. Recall that for a group $G$, a subgroup $H<G$ is called characteristic if for every isomorphism $f: G \rightarrow G$, we get $f(H)=H . H_{123}$ is a characteristic subgroup and hence every homeomorphism on $F_{\min }$ lifts to the covering $F_{123}$ that corresponds to $H_{123}<\pi_{1}\left(F_{\min }\right)$. Since each $\phi_{i}: F_{i} \rightarrow F_{i}$ is a lift of $\phi_{\text {min }}^{l_{i}}$, it can be lifted to $\widetilde{\phi}_{i}: F_{123} \rightarrow F_{123}$. Let $l$ be the least common multiple of $l_{i}$ 's, then by putting $k_{i}=l / l_{i}$, we get $\widetilde{\phi}_{1}^{k_{1}}=\widetilde{\phi}_{2}^{k_{2}}=\widetilde{\phi}_{3}^{k_{3}}$ on $F_{123}$.

Remark 2.12. We do not know if the transitivity or the equivalence of fibered commensurability and commensurability in the sense of Definition 1.2 holds for the case where the automorphisms are periodic or reducible.

\section{Thurston norm and normalized entropy}

Thurston norm. Let $M$ be a fibered hyperbolic 3-manifold. In this subsection we recall briefly the Thurston norm on $H^{1}(M ; \mathbb{R})$ and discuss the relationship between fibered commensurability of fibrations on a fixed manifold $M$ and the normalized entropy. For more details about the Thurston norm, see [Thurston 1986; Kapovich 2001; Kin and Takasawa 2011]. For any (possibly disconnected) compact surface $F=F_{1} \sqcup F_{2} \sqcup \cdots \sqcup F_{n}$, let $\chi_{-}(F)$ be the sum of the absolute values of Euler characteristics $\left|\chi\left(F_{i}\right)\right|$ of components with negative Euler characteristics. For a given $\omega \in H^{1}(M ; \mathbb{Z}) \subset H^{1}(M ; \mathbb{R})$, we define $\|\omega\|$ to be

$\min \left\{\chi_{-}(F) \mid F\right.$ is an embedded orientable surface $(F, \partial F) \subset(M, \partial M)$, and

$$
\left.[F] \in H_{2}(M, \partial M ; \mathbb{Z}) \text { is the Poincaré dual of } \omega \in H^{1}(M ; \mathbb{Z})\right\} \text {. }
$$

If $F$ realizes the minimum, we call $F$ a minimal representative of $\omega$. We can extend this norm to $H^{1}(M ; \mathbb{Q})$ by $\|\omega\|=\|r \omega\| / r$. It turns out that $\|\cdot\|$ extends continuously to $H^{1}(M ; \mathbb{R})$. Further, this $\|\cdot\|$ turns out to be seminorm on $H^{1}(M ; \mathbb{R})$ and the unit ball $U=\left\{\omega \in H^{1}(M ; \mathbb{R}) \mid\|\omega\| \leq 1\right\}$ is a compact convex polygon [Thurston 1986]. The seminorm $\|\cdot\|$ is called the Thurston norm on $H^{1}(M ; \mathbb{R})$. We need some more terminologies to explain the relationship between $\|\cdot\|$ and fibrations on $M$. We denote

- the cone over a top-dimensional face $\Delta$ of the unit ball $U$ by $C_{\Delta}$,

- the set of integral classes on $\operatorname{Int}\left(C_{\Delta}\right)$ by $\operatorname{Int}\left(C_{\Delta}(\mathbb{Z})\right)$, and

- the set of rational classes on a top-dimensional face $\Delta$ by $\Delta(\mathbb{Q})$.

Theorem 3.1 [Thurston 1986]. Let $M$ be a fibered hyperbolic 3-manifold and $F$ the fiber. Then there is a top-dimensional face $\Delta$ of $U$ such that 
- the dual of $[F] \in H_{2}(M, \partial M ; \mathbb{Z})$ belongs to $\operatorname{Int}\left(C_{\Delta}(\mathbb{Z})\right)$, and

- for every primitive class $\omega$ in $\operatorname{Int}\left(C_{\Delta}(\mathbb{Z})\right)$, a minimal representative of $\omega$ is the fiber of a fibration on $M$.

We call the face $\Delta$ in Theorem 3.1 a fibered face and the cone over a fibered face a fibered cone.

As a corollary, we see that if the first Betti number $b_{1}(M)>1$ and $M$ is fibered, then $M$ has infinitely many distinct fibrations. We will discuss fibered commensurability of fibrations of a hyperbolic fibered 3-manifold.

Normalized entropy. The normalized entropy is shared by commensurable fibrations on a fixed hyperbolic 3-manifold.

Proposition 3.2. Suppose that $\left[F_{1}, \phi_{1}\right]=\left[F_{2}, \phi_{2}\right]$ and their interior admit hyperbolic metrics. If $\left(F_{1}, \phi_{1}\right)$ is commensurable to $\left(F_{2}, \phi_{2}\right)$, then

$$
\chi\left(F_{1}\right) \log \lambda\left(\phi_{1}\right)=\chi\left(F_{2}\right) \log \lambda\left(\phi_{2}\right) .
$$

Proof. There are pairs $\left(\widetilde{F}, \tilde{\phi}_{i}\right)$ that cover $\left(F_{i}, \phi_{i}\right)$ and $k_{i} \in \mathbb{Z} \backslash\{0\}$ for $i=1,2$ such that $\widetilde{\phi}_{1}^{k_{1}}=\widetilde{\phi}_{2}^{k_{2}}$. Then the mapping torus $\left[\widetilde{F}, \widetilde{\phi}_{i}^{k_{i}}\right]$ covers $\left[F_{i}, \phi_{i}\right]$ and the degree of this cover is $k_{i} \chi(\tilde{F}) / \chi\left(F_{i}\right)$. Since $\left[F_{1}, \phi_{1}\right]=\left[F_{2}, \phi_{2}\right]$, we get $k_{1} / \chi\left(F_{1}\right)=$ $k_{2} / \chi\left(F_{2}\right)$. Since $\lambda(\phi)=\lambda(\tilde{\phi})$,

$$
\chi(\tilde{F}) \log \lambda\left(\tilde{\phi}_{i}^{k_{i}}\right)=\frac{\chi(\tilde{F})}{\chi\left(F_{1}\right)} \chi\left(F_{1}\right) k_{1} \log \lambda\left(\phi_{1}\right)=\frac{\chi(\tilde{F})}{\chi\left(F_{2}\right)} \chi\left(F_{2}\right) k_{2} \log \lambda\left(\phi_{2}\right) .
$$

Putting them all together, we get $\chi\left(F_{1}\right) \log \lambda\left(\phi_{1}\right)=\chi\left(F_{2}\right) \log \lambda\left(\phi_{2}\right)$.

Each primitive integral class in $C_{\Delta}(\mathbb{Z})$ corresponds to a rational class in $\operatorname{Int}(\Delta)$. The normalized entropy defines a function ent $: \Delta(\mathbb{Q}) \rightarrow \mathbb{R}$. In [Fried 1982], the function $1 /$ ent is shown to be concave and therefore it extends to $\operatorname{Int}(\Delta)$. Moreover:

Theorem 3.3 [McMullen 2000]. The function $1 /$ ent $: \operatorname{Int}(\Delta) \rightarrow \mathbb{R}$ is strictly concave.

In Example 3.12 of [Calegari et al. 2011], it is remarked that some fibrations on $S^{3} \backslash 6_{2}^{2}$ are not commensurable. In Corollary 2.8, it is proved that all fibrations on $S^{3} \backslash 6_{2}^{2}$ are minimal elements and since each minimal element is unique, we see that two fibrations of $S^{3} \backslash 6_{2}^{2}$ are either symmetric or noncommensurable. Here, we give an alternative proof of this fact in terms of the normalized entropy. In [Hironaka 2010; McMullen 2000], the unit ball of the Thurston norm on $H^{1}\left(S^{3} \backslash 6_{2}^{2}\right)$ is computed to be a square. Further, the symmetries of the square all come from the symmetries of the manifold (see Example 4.5 for more details about the symmetries of $S^{3} \backslash 6_{2}^{2}$ ). Therefore the function 1 /ent is invariant under the action of the symmetries of the unit ball. Since $1 /$ ent is strictly concave, this proves that any two fibrations that correspond to distinct elements in $H^{1}(M ; \mathbb{Z})$ are either symmetric 
or noncommensurable. In other words, the normalized entropy determines the commensurability class of a fibration on $S^{3} \backslash 6_{2}^{2}$ up to symmetry.

On the other hand, in [Kin et al. 2012, §2], it is observed that for the magic 3-manifold $N$ there are rational points on a fibered face which share the same normalized entropy but which are not symmetric to each other. However, again by Corollary 2.8, we also see that any two distinct fibrations of $N$ are either symmetric or noncommensurable. Hence for the magic 3-manifold, the commensurability classes of fibrations are not determined by the normalized entropies. We do not know for what kind of hyperbolic 3-manifolds the commensurability classes of fibrations on the same hyperbolic 3-manifold are determined by the normalized entropy up to symmetry.

\section{Commensurability of fibrations on a hyperbolic 3-manifold}

In this section we prove Theorems 1.4 and 1.3.

Manifolds without hidden symmetries. We start with some definitions. A Kleinian group is a discrete subgroup of $\operatorname{PSL}(2, \mathbb{C})$. Two Kleinian groups $\Gamma_{1}$ and $\Gamma_{2}$ are said to be commensurable if $\Gamma_{1} \cap \Gamma_{2}$ is a finite-index subgroup of both $\Gamma_{1}$ and $\Gamma_{2}$. Let $\Gamma$ be a Kleinian group. The commensurator $C^{+}(\Gamma)$ of $\Gamma$ is

$$
C^{+}(\Gamma)=\left\{h \in \operatorname{PSL}(2, \mathbb{C}) \mid \Gamma \text { and } h \Gamma h^{-1} \text { are commensurable }\right\},
$$

and the normalizer $N^{+}(\Gamma)$ is

$$
N^{+}(\Gamma)=\left\{h \in \operatorname{PSL}(2, \mathbb{C}) \mid \Gamma=h \Gamma h^{-1}\right\} .
$$

Note that $N^{+}(\Gamma)<C^{+}(\Gamma)$.

Let $M$ be a hyperbolic 3-manifold and $\rho: \pi_{1}(M) \rightarrow \Gamma<\operatorname{PSL}(2, \mathbb{C})$ a holonomy representation of $\pi_{1}(M)$. By the Mostow-Prasad rigidity theorem, any self-homeomorphism $\varphi: M \rightarrow M$ corresponds to a conjugation of $\Gamma$. Therefore we get $N(\Gamma) / \Gamma \cong \operatorname{Isom}(M)$, where $\operatorname{Isom}(M)$ is the group of self-homeomorphisms of $M$. If $C^{+}(\Gamma) \backslash N^{+}(\Gamma) \neq \varnothing$, each nontrivial element $h \in C^{+}(\Gamma) \backslash N^{+}(\Gamma)$ is said to be a hidden symmetry. Then $M$ is said to have no hidden symmetries if $\Gamma$ has no hidden symmetries. Note that by the Mostow-Prasad rigidity theorem, the holonomy representations of $\pi_{1}(M)$ are related by a conjugation. Hence the definition does not depend on the choice of a holonomy representation.

Proof of Theorem 1.4. Let $\left(M, \mathscr{F}_{1}\right)$ and $\left(M, \mathscr{F}_{2}\right)$ be commensurable fibered pairs that correspond to two distinct fibrations on $M$. By Theorem 2.6 we have a unique minimal element $(N, \mathscr{G})$ in the commensurability class. Let $\rho: \pi_{1}(N) \rightarrow$ $\operatorname{PSL}(2, \mathbb{C})$ be a holonomy representation and $\Gamma:=\rho\left(\pi_{1}(N)\right)$. Since $\left(M, \mathscr{F}_{1}\right)$ and $\left(M, \mathscr{F}_{2}\right)$ cover $(N, \mathscr{G})$, there are two corresponding coverings $p_{1}, p_{2}: M \rightarrow N$. 
Let $\Gamma_{i}=\rho p_{i *}\left(\pi_{1}(M)\right)$ for $i=1,2$. By the Mostow-Prasad rigidity theorem, there is $h \in \operatorname{PSL}(2, \mathbb{C})$ such that $h \Gamma_{1} h^{-1}=\Gamma_{2}$. Further, since $\Gamma_{2}<\Gamma \cap h \Gamma h^{-1}$, $h \in C^{+}(\Gamma)=C^{+}\left(\Gamma_{1}\right)=N^{+}\left(\Gamma_{1}\right)$. The last equality holds since $M$ has no hidden symmetries. It follows that $\Gamma_{1}=\Gamma_{2}$ and hence there exists a homeomorphism $\varphi: M \rightarrow M$ such that $p_{1} \varphi=p_{2}$. Therefore $\omega_{1}$ and $\omega_{2}$ are symmetric.

Remark 4.1. Hyperbolic 3-manifolds with hidden symmetries are "rare" among all nonarithmetic hyperbolic 3-manifolds (see for example, [Goodman et al. 2008]). Hence we may expect that "most" hyperbolic 3-manifolds have no hidden symmetries and therefore have no nonsymmetric but commensurable fibration.

Remark 4.2. As mentioned above, there are no nonsymmetric but commensurable fibrations on $S^{3} \backslash 6_{2}^{2}$ and the magic 3-manifold. However, $S^{3} \backslash 6_{2}^{2}$ and the magic 3-manifold are arithmetic and by a result of Margulis [1991], they have lots of hidden symmetries. Therefore even though a manifold has hidden symmetries, it might not have any nonsymmetric but commensurable fibrations.

Nonsymmetric and commensurable fibrations. We now prove Theorem 1.3 by constructing examples of manifolds that have nonsymmetric but commensurable fibrations.

Lemma 4.3. Let $M$ be a fibered hyperbolic 3-manifold. Suppose two primitive elements $\omega_{1} \neq \pm \omega_{2} \in H^{1}(M ; \mathbb{Z})$ correspond to fibrations with the fibers and the monodromies $\left(F_{1}, \phi_{1}\right)$ and $\left(F_{2}, \phi_{2}\right)$ respectively. We suppose further $\left(F_{1}, \phi_{1}\right)=$ $\left(F_{2}, \phi_{2}\right)$ (that is, conjugate to each other). Then, for all large enough $n \in \mathbb{N}$, there exists a degree $n$ covering space $p_{n}: M_{n} \rightarrow M$ such that $p_{n}^{*}\left(\omega_{1}\right)$ and $p_{n}^{*}\left(\omega_{2}\right)$ correspond to commensurable but nonsymmetric fibrations.

Proof. Note that by the universal coefficient theorem, we have

$$
H^{1}(M ; \mathbb{Z}) \cong \operatorname{Hom}\left(H_{1}(M) / \operatorname{Tor}, \mathbb{Z}\right),
$$

where Tor is the torsion part. This isomorphism is determined by a choice of a basis of $H_{1}(M ; \mathbb{Z}) /$ Tor. Let $A_{i}=\mathrm{ab}\left(\pi_{1}\left(F_{i}\right)\right) /$ Tor, where ab: $\pi_{1}(M) \rightarrow H_{1}(M)$ is the abelianization and $\pi_{1}\left(F_{i}\right) \hookrightarrow \pi_{1}(M)$ is an injection induced by the fiber bundle structure of $M$ associated to $\left(F_{i}, \phi_{i}\right)$ for $i=1,2$. The fiber bundle structure of $M$ gives the exact sequence

$$
0 \rightarrow \pi_{1}\left(F_{i}\right) \rightarrow \pi_{1}(M) \stackrel{\rho_{i}}{\rightarrow} \pi_{1}\left(S^{1}\right) \cong \mathbb{Z} \rightarrow 0 .
$$

The map $\rho_{i}$ factors through the abelianization since $\pi_{1}\left(S^{1}\right) \cong \mathbb{Z}$ is abelian. Hence we get $A_{i}=\operatorname{Ker}\left(\omega_{i}\right) \cong \mathbb{Z}^{b-1}$, where $b$ is the first Betti number of $M$. We consider the dynamical covering $p_{n}: M_{n} \rightarrow M$ of degree $n$ with respect to $\omega_{1}$ (that is, the covering corresponding to $\left.\left(F_{1}, \phi_{1}^{n}\right)\right)$. This is the covering corresponding to the 

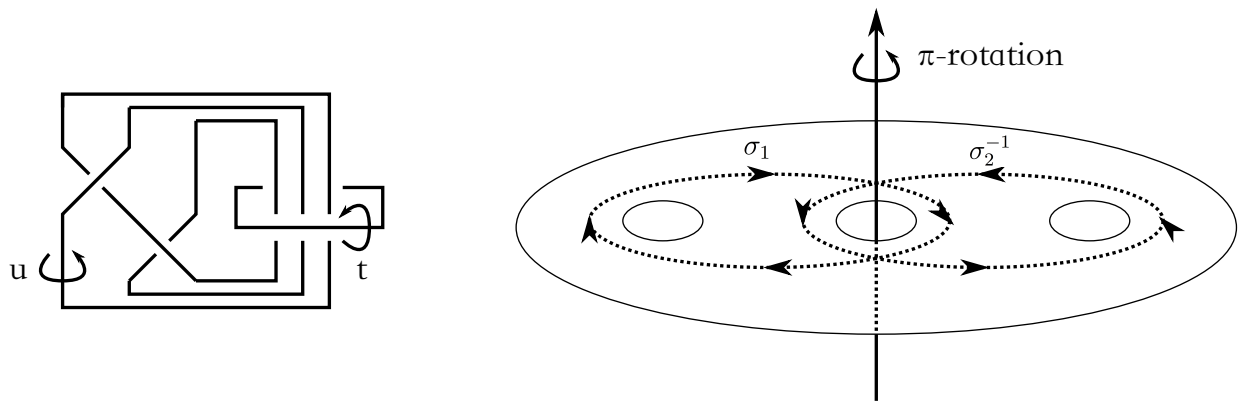

Figure 3. An involution map $h$ on the 4-holed sphere.

surjective map

$$
\pi_{1}(M) \stackrel{\mathrm{ab}}{\rightarrow} H_{1}(M) \stackrel{\omega_{1}}{\rightarrow} \mathbb{Z} \rightarrow \mathbb{Z} / n \mathbb{Z} .
$$

For sufficiently large $n$, there exists $a \in A_{2}$ such that $a$ maps to a nonzero element by the above surjective map. This means that each component of $p_{n}^{-1}\left(F_{2}\right)$ is not homeomorphic to $F_{2}$.

Example 4.4. The 3-manifold $S^{3} \backslash 6_{2}^{2}$ and the magic manifold have symmetries that permute cusps, and therefore they do have two distinct elements in their first cohomology with homeomorphic fibers and conjugate monodromies.

Example 4.5. In this example we observe that $M:=S^{3} \backslash 6_{2}^{2}$ has two symmetric fibrations in the same fibered cone in $H^{1}(M)$. Although this fact can be checked by computing the symmetry group by SnapPy [Culler et al. 2013], we give a geometric proof. The first half of the following argument is due to Eriko Hironaka, see also [Hironaka 2010].

Let $u, t$ be the generators of $H_{1}(M, \mathbb{Z})$ that correspond to the meridians of $6_{2}^{2}$ (see the left picture of Figure 3). Let $U, T \in H^{1}(M ; \mathbb{Z})$ be the dual of $u, t$ respectively. Then $U$ corresponds to the fibration of $M$ with monodromy $f$ that corresponds to $\sigma_{1} \sigma_{2}^{-1} \in B_{3}$. Let $h$ be a $\pi$-rotation, which is depicted in Figure 3 . We can see that $f^{-1}=\sigma_{2} \sigma_{1}^{-1}=h f h$, that is $f$ and $f^{-1}$ are conjugate to each other. Then we take the mirror image of $6_{2}^{2}$. By isotopy and above conjugacy, we see that $6_{2}^{2}$ is amphicheiral. The induced map on $H^{1}(M ; \mathbb{Z})$ of the symmetry on $M$ that gives amphicheirality satisfies $U \mapsto-U$ and $T \mapsto T$. This symmetry preserves the fibered face $\Delta:=\{a U+b T \mid-1<a<1, b=1\}$. By this symmetry, we see that fibrations on the cone $C_{\Delta}$ over $\Delta$ of the form $n U+m T$ and $-n U+m T(n, m \in \mathbb{Z})$ are symmetric.

Proof of Theorem 1.3. Putting Lemma 4.3 and Example 4.5 together, we have a proof. 


\section{Acknowledgments}

I would like to thank Sadayoshi Kojima and members of his research group for helpful conversations and advice. I would also like to thank Eriko Hironaka for her inspiring series of lectures about pseudo-Anosov maps at Tokyo Tech and for telling me the argument in Example 4.5. I would also like to thank Neil Hoffman, Tamás Kálmán, and Hongbin Sun for catching some errors in an earlier version of this paper. Finally I would like to thank the anonymous referee for helpful suggestions.

\section{References}

[Agol 2010] I. Agol, "The minimal volume orientable hyperbolic 2-cusped 3-manifolds", Proc. Amer. Math. Soc. 138:10 (2010), 3723-3732. MR 2011k:57023 Zbl 1203.57006

[Calegari et al. 2011] D. Calegari, H. Sun, and S. Wang, "On fibered commensurability", Pacific J. Math. 250:2 (2011), 287-317. MR 2012d:57029 Zbl 1236.57022

[Cao and Meyerhoff 2001] C. Cao and G. R. Meyerhoff, "The orientable cusped hyperbolic 3manifolds of minimum volume”, Invent. Math. 146:3 (2001), 451-478. MR 2002i:57016 Zbl 1028. 57010

[Carlson 2010] J. D. Carlson, "Commensurability of two-multitwist pseudo-Anosovs", preprint, 2010. arXiv 1011.0247

[Casson and Bleiler 1988] A. J. Casson and S. A. Bleiler, Automorphisms of surfaces after Nielsen and Thurston, London Mathematical Society Student Texts 9, Cambridge University Press, 1988. MR 89k:57025 Zbl 0649.57008

[Culler et al. 2013] M. Culler, N. M. Dunfield, and J. R. Weeks, "SnapPy: a computer program for studying the geometry and topology of 3-manifolds", 2013, http://snappy.computop.org.

[Fried 1982] D. Fried, "Flow equivalence, hyperbolic systems and a new zeta function for flows", Comment. Math. Helv. 57:2 (1982), 237-259. MR 84g:58083 Zbl 0503.58026

[Gehring et al. 1998] F. W. Gehring, C. Maclachlan, and G. J. Martin, "Two-generator arithmetic Kleinian groups, II”, Bull. London Math. Soc. 30:3 (1998), 258-266. MR 99j:30050 Zbl 0930.30037

[Goodman et al. 2008] O. Goodman, D. Heard, and C. Hodgson, "Commensurators of cusped hyperbolic manifolds", Experiment. Math. 17:3 (2008), 283-306. MR 2009h:57029 Zbl 05500548

[Hironaka 2010] E. Hironaka, "Small dilatation mapping classes coming from the simplest hyperbolic braid”, Algebr. Geom. Topol. 10:4 (2010), 2041-2060. MR 2012e:57033 Zbl 1221.57028

[Ivanov 1988] N. V. Ivanov, “Коэффициенты растяжения псевдоаносовских гомеоморфизмов", Zap. Nauchn. Sem. (LOMI) 167 (1988), 111-116. Translated as "The stretching coefficients of pseudo-Anosov homeomorphisms" in Journal of Soviet Mathematics 52:1 (1990), 2819-2822. MR 89i:32047 Zbl 0693.57007

[Kapovich 2001] M. Kapovich, Hyperbolic manifolds and discrete groups, Progress in Mathematics 183, Birkhäuser, Boston, 2001. MR 2002m:57018 Zbl 0958.57001

[Kin and Takasawa 2011] E. Kin and M. Takasawa, "Pseudo-Anosov braids with small entropy and the magic 3-manifold”, Comm. Anal. Geom. 19:4 (2011), 705-758. MR 2880213 Zbl 1251.37047

[Kin et al. 2012] E. Kin, S. Kojima, and M. Takasawa, "Minimal dilatations of pseudo-Anosovs generated by the magic 3-manifold and their asymptotic behavior", preprint, 2012. arXiv 1104.3939

[Margulis 1991] G. A. Margulis, Discrete subgroups of semisimple Lie groups, Ergebnisse der Mathematik und ihrer Grenzgebiete (3) 17, Springer, Berlin, 1991. MR 92h:22021 Zbl 0732.22008 
[McMullen 2000] C. T. McMullen, "Polynomial invariants for fibered 3-manifolds and Teichmüller geodesics for foliations", Ann. Sci. École Norm. Sup. (4) 33:4 (2000), 519-560. MR 2002d:57015 Zbl 1013.57010

[Otal 1996] J.-P. Otal, Le théorème d'hyperbolisation pour les variétés fibrées de dimension 3, Astérisque 235, Soc. Math. France, Paris, 1996. Translated as The hyperbolization theorem for fibered 3-manifolds, SMF/AMS Texts and Monographs 7, Amer. Math. Soc, Providence, RI, 2001. MR 97e:57013 Zbl 0855.57003

[Thurston 1979] W. P. Thurston, "The geometry and topology of three-manifolds", lecture notes, Princeton University, 1979, http://msri.org/publications/books/gt3m.

[Thurston 1986] W. P. Thurston, "A norm for the homology of 3-manifolds", pp. 99-130 Mem. Amer. Math. Soc. 339, Amer. Math. Soc., Providence, RI, 1986. The full issue including this paper is available at http://dx.doi.org/10.1090/memo/0339. MR 88h:57014 Zbl 0585.57006

[Thurston 1988] W. P. Thurston, "On the geometry and dynamics of diffeomorphisms of surfaces", Bull. Amer. Math. Soc. (N.S.) 19:2 (1988), 417-431. MR 89k:57023 Zbl 0674.57008

[Walsh 2011] G. S. Walsh, "Orbifolds and commensurability”, pp. 221-231 in Interactions between hyperbolic geometry, quantum topology and number theory, edited by A. Champagnerkar et al., Contemp. Math. 541, Amer. Math. Soc., Providence, RI, 2011. MR 2012e:57048 Zbl 1231.57017

Received October 24, 2012. Revised July 2, 2013.

\author{
Hidetoshi MASAI \\ Department of Mathematical and Computing SCiEnCES \\ TOKYO INSTITUTE OF TECHNOLOGY \\ 2-12-1 O-OKAYAMA, MEgURO-KU \\ TOKYO 152-8552 \\ JAPAN \\ masai9@is.titech.ac.jp
}




\title{
PACIFIC JOURNAL OF MATHEMATICS
}

\author{
msp.org/pjm
}

Founded in 1951 by E. F. Beckenbach (1906-1982) and F. Wolf (1904-1989)

\section{EDITORS}

V. S. Varadarajan (Managing Editor)

Department of Mathematics

University of California

Los Angeles, CA 90095-1555

pacific@math.ucla.edu

Paul Balmer

Department of Mathematics

University of California

Los Angeles, CA 90095-1555

balmer@math.ucla.edu

Daryl Cooper

Department of Mathematics

University of California

Santa Barbara, CA 93106-3080 cooper@math.ucsb.edu

Jiang-Hua Lu

Department of Mathematics

The University of Hong Kong

Pokfulam Rd., Hong Kong jhlu@maths.hku.hk
Don Blasius

Department of Mathematics University of California

Los Angeles, CA 90095-1555

blasius@math.ucla.edu

Robert Finn

Department of Mathematics Stanford University

Stanford, CA 94305-2125

finn@math.stanford.edu

Sorin Popa

Department of Mathematics

University of California

Los Angeles, CA 90095-1555 popa@math.ucla.edu

Paul Yang

Department of Mathematics Princeton University

Princeton NJ 08544-1000

yang@math.princeton.edu

\section{PRODUCTION}

Silvio Levy, Scientific Editor, production@msp.org

\section{SUPPORTING INSTITUTIONS}

ACADEMIA SINICA, TAIPEI

CALIFORNIA INST. OF TECHNOLOGY

INST. DE MATEMÁTICA PURA E APLICADA

KEIO UNIVERSITY

MATH. SCIENCES RESEARCH INSTITUTE

NEW MEXICO STATE UNIV.

OREGON STATE UNIV.

\author{
STANFORD UNIVERSITY \\ UNIV. OF BRITISH COLUMBIA \\ UNIV. OF CALIFORNIA, BERKELEY \\ UNIV. OF CALIFORNIA, DAVIS \\ UNIV. OF CALIFORNIA, LOS ANGELES \\ UNIV. OF CALIFORNIA, RIVERSIDE \\ UNIV. OF CALIFORNIA, SAN DIEGO \\ UNIV. OF CALIF., SANTA BARBARA
}

\author{
Vyjayanthi Chari \\ Department of Mathematics \\ University of California \\ Riverside, CA 92521-0135 \\ chari@math.ucr.edu \\ Kefeng Liu \\ Department of Mathematics \\ University of California \\ Los Angeles, CA 90095-1555 \\ liu@math.ucla.edu \\ Jie Qing \\ Department of Mathematics \\ University of California \\ Santa Cruz, CA 95064 \\ qing@cats.ucsc.edu
}

These supporting institutions contribute to the cost of publication of this Journal, but they are not owners or publishers and have no responsibility for its contents or policies.

See inside back cover or msp.org/pjm for submission instructions.

The subscription price for 2013 is US \$400/year for the electronic version, and \$485/year for print and electronic.

Subscriptions, requests for back issues and changes of subscribers address should be sent to Pacific Journal of Mathematics, P.O. Box 4163, Berkeley, CA 94704-0163, U.S.A. The Pacific Journal of Mathematics is indexed by Mathematical Reviews, Zentralblatt MATH, PASCAL CNRS Index, Referativnyi Zhurnal, Current Mathematical Publications and Web of Knowledge (Science Citation Index).

The Pacific Journal of Mathematics (ISSN 0030-8730) at the University of California, c/o Department of Mathematics, 798 Evans Hall \#3840, Berkeley, CA 94720-3840, is published twelve times a year. Periodical rate postage paid at Berkeley, CA 94704, and additional mailing offices. POSTMASTER: send address changes to Pacific Journal of Mathematics, P.O. Box 4163, Berkeley, CA 94704-0163.

PJM peer review and production are managed by EditFLOW ${ }^{\circledR}$ from Mathematical Sciences Publishers.

\section{PUBLISHED BY}

mathematical sciences publishers

nonprofit scientific publishing

http://msp.org/

(C) 2013 Mathematical Sciences Publishers 


\section{PACIFIC JOURNAL OF MATHEMATICS}

Volume $266 \quad$ No. $2 \quad$ December 2013

Rate of attraction for a semilinear wave equation with variable coefficients and

critical nonlinearities

FÁGNER DiAs ARARUNA and FLANK DAVID MORAIS BEZERRA

The Brin-Thompson groups $s V$ are of type $\mathrm{F}_{\infty}$

Martin G. Fluch, Marco Marschler, Stefan Witzel and

MATTHEW C. B. ZAREMSKY

Ideal decompositions of a ternary ring of operators with predual

MASAYOSHI KANEDA

A study of real hypersurfaces with Ricci operators in 2-dimensional complex space 305 forms

Dong Ho Lim, WoOn Ha SoHn and Hyunjung Song

On commensurability of fibrations on a hyperbolic 3-manifold

HideTOSHI MASAI

Multiplicative Dirac structures

CRISTIÁN ORTIZ

On the finite generation of a family of Ext modules

TONY J. PuthenPuRAKAL

Index formulae for Stark units and their solutions

XAVIER-FranÇOIS ROBLOT

The short time asymptotics of Nash entropy

GUOYI XU

Several splitting criteria for vector bundles and reflexive sheaves

STEPHEN S.-T. YAU and FEI YE

The minimal volume orientable hyperbolic 3-manifold with 4 cusps

KEN'ICHI YOSHIDA

On the Witten rigidity theorem for string ${ }^{c}$ manifolds

JIANQING YU and BO LIU 